\title{
Maintaining Communication Between an Explorer and a Base Station ${ }^{\star}$
}

\author{
Miroslaw Dynia ${ }^{1}$, Jarosław Kutyłowski ${ }^{2}$, Paweł Lorek ${ }^{3}$, \\ and Friedhelm Meyer auf der Heide ${ }^{4}$ \\ 1 DFG Graduate College "Automatic Configuration in Open Systems", \\ Heinz Nixdorf Institute, University of Paderborn \\ 2 International Graduate School, Heinz Nixdorf Institute, University of Paderborn \\ 3 Mathematical Institute, Wrocław University \\ ${ }^{4}$ Heinz Nixdorf Institute, University of Paderborn
}

\begin{abstract}
Consider a (robotic) explorer starting an exploration of an unknown terrain from its base station. As the explorer has only limited communication radius, it is necessary to maintain a line of robotic relay stations following the explorer, so that consecutive stations are within the communication radius of each other. This line has to start in the base station and to end at the explorer.

In the simple scenario considered here we assume an obstacle-free terrain, so that the shortest connection (the one which needs the smallest number of relay stations) is a straight line. We consider an explorer who goes an arbitrary, typically winding way, and define a very simple, intuitive, fully local, distributed strategy for the relay stations our Go-To-THE-MiddLe strategy - to maintain a line from the base station to the robot as short as possible.
\end{abstract}

Besides the definition of this strategy, we present an analysis of its performance under different assumptions. For the static case we prove a bound on the convergence speed, for the dynamic case we present experimental evaluations that show the quality of our strategy under different types of routes the explorer could use.

\section{Introduction}

In our research we investigate the exploration of a planar terrain without obstacles. To achieve this goal, an explorer is used who starts its work at a base station and progresses to gather information about the whole terrain. In order to construct a communication path between this explorer and the base station, we employ mobile relay stations. These relay stations are small, mobile robots

\footnotetext{
* Partially supported by the EU within the 6th Framework Programme under contract 001907 (DELIS) and by the DFG-Sonderforschungsbereich SPP 1183: "Organic Computing. Smart Teams: Local, Distributed Strategies for Self-Organizing Robotic Exploration Teams".
}

Please use the following format when citing this chapter:

Dynia, M., Kutylowski, J., Lorek, P., auf der Heide, F.M., 2006, in IFIP International Federation for Information Processing, Volume 216, Biologically Inspired Cooperative Computing, eds. Pan, Y., Rammig, F., Schmeck, H., Solar, M, (Boston: Springer), pp. 137-146. 
which are responsible for routing messages between the explorer and the base station.

In order to minimize the number of necessary relay stations, they should be organized on a line close to the straight line connecting the explorer and the base station. Furthermore it is necessary that the relay stations can communicate with each other, so consecutive stations must be placed in a limited distance on this line.

Since we allow the explorer to walk along an arbitrary route, its position updates frequently. Arranging all relay stations on the straight line would thus require to communicate its position updates to the whole path, resulting in a globally controlled strategy incuring a substantial communication load. Thus we are looking for simple distributed, local strategies which allow the relay stations to arrange near to their optimal positions based on very local information. We introduce a strategy, our Go-To-ThE-MiddeE, which does not use any communication - relay stations perform their movement basing only on sensed positions of their communication partners. This approach is called "interaction via sensing" as defined in [1].

In Section 2 we introduce a local and distributed strategy which keeps the relay stations close to their optimal positions on the line. This strategy is very intuitive and could also be used by human explorers. A similar behavior can be observed in bird flocks maintaining formation (see [2]).

We analyze our strategy both in a static and in a dynamic setting. In Section 3 we describe the static setting, where the explorer does not move, and the relay stations are initially placed on an arbitrarily winding route taken by the explorer until now. We give a worst-case theoretical analysis which describes the time needed for the relay stations to converge to positions near the straight line between the base station and the explorer. In Section 4 we let the explorer move and let the relay stations continuously apply our strategy. We experimentally evaluate the performance of the strategy, using three different types of routes taken by the explorer. The proofs of several technical lemmas can be found in the full version of this paper.

\subsection{Related work}

From a general point of view, our work can be positioned in the area of swarm intelligence [3], particularly in the field of robotic intelligent swarms $[4,5,1]$.

Our work has much in common with the prior research in the areas of pattern formation and formation maintenance. The work described in $[6,7,8,9,10]$ considers swarms of robots which should self-organize to form a pattern (a line, circle, ...) on a plane or to maintain a formation while marching.

The most similar work to ours is [6]. Among others it presents an algorithm CONTRACTION which is very similar to our strategy. Nevertheless, although the topic of forming a geometric pattern on a plane has been considered very often, we are not aware of any analysis giving strong theoretical bounds on the worstcase performance of a strategy. Up to our knowledge the topic of mobile robots 
self-organizing to form a line has not been evaluated experimentally under the performance aspect yet.

\subsection{Model}

We construct a graph modeling the base station, the explorer and the relay stations with vertices. The vertices are always logically organized in a path $\left(v_{1}, v_{2}, \ldots, v_{n-1}, v_{n}\right)$, where $v_{1}$ corresponds to the base station, $v_{n}$ to the explorer and $v_{2}, \ldots, v_{n-1}$ to the relay stations. To represent the path we introduce undirected edges $\left(v_{i}, v_{i+1}\right)$ for every $i \in\{1, \ldots, n-1\}$. The communication is routed along this path from $v_{1}$ to $v_{n}$ or in the other direction. The graph is embedded on a plane, thus we will use the notion of a position $p(v)$ of a vertex $v$. Distances between vertices are given by the $L_{2}$ norm and described by $\left|\left(v_{i}, v_{i+1}\right)\right|$.

The goal of a strategy minimizing the distance between the relay stations is to arrange the relay stations on the line between $v_{1}$ and $v_{n}$ in equal distances from each other, or, in other words, to bring the relay stations as near to this optimal positions as possible.

We require every edge on the path $v_{1}, \ldots, v_{n}$ to have at most length $d$, so that the maximum transmission distance of $d$ is not exceed and communication links between partners on the communication path can be hold up. A communication path fulfilling this property is called valid.

\section{The Go-To-The-Middle Strategy}

The following GO-TO-THE-MIDDLE strategy is executed repeatedly by every relay station. Relay station $i$ observes the positions $p\left(v_{i-1}\right)$ and $p\left(v_{i+1}\right)$ of its communication partners and moves itself into the middle of the interval from $v_{i-1}$ to $v_{i+1}$.

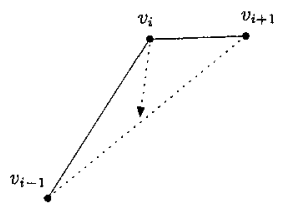

Fig. 1. Node $v_{i}$ executes Go-To-The-Midde strategy by moving into the middle of the interval between $v_{i-1}$ and $v_{i+1}$.

For simplification of the analysis we will assume that the Go-To-THEMIDDLE strategy is invoked in discrete time steps. Each time step is subdivided into two shorter substeps. In the first one, all relay stations check the positions 
of their neighbors. In the second substep all relay stations move to the middle of the observed positions of its neighbors as described above.

Since the explorer moves, it may be necessary to extend the path of relay stations. We perform this at the end of the path, between the last relay station and the explorer. This happens every time the distance between $v_{n-1}$ and $v_{n}$ increases to more than $d$. We rename the vector $v$ appropriately, so that $v_{n+1}$ describes the explorer and $v_{n}$ the new relay station. The new relay station is inserted in the middle of the interval connecting the last relay station and the explorer.

If the explorer can carry a sufficiently large pool of relay stations then this strategy is easily executed, since new relay stations are available at the explorer's position.

If this is not the case, the base station has to make sure that enough relay stations are on the path. As it does not know about the position and movement of the explorer, we modify the strategy slightly. We add to each relay station $v_{i}$ a second one, its partner, at the same position as $v_{i}$. During each Go-ToTHE-MIDDLE step, a relay station and its partner perform the same movement. Afterward, one of them goes the next relay station and becomes its partner. At the base station, a new relay station is introduced as the partner of $v_{2}$. At the explorer there are two possibilities. If the explorer has not moved far away from $v_{n}$, the partner of $v_{n}$ starts going back to the base station and is reused there. If a new relay station is needed to hold up the communication with the explorer, the partner of $v_{n}$ is used. Limiting the maximal speed of the explorer to $d / 2$ it will be necessary to insert a new relay station to the communication path at most every two rounds. Thus the new relay station in the communication path will obtain its partner in the next round after it has been inserted.

This modified strategy uses at most $2 n$ relay station in addition to those needed by Go-TO-THE-MIDDLE, half of them being the partners of all relay stations and half of them being on their way back to the base station.

We may also consider removing relay stations when they are close enough to each other. Formally, a relay station $v_{i}$ can be removed from the path if the distance $\left|\left(v_{i-1}, v_{i+1}\right)\right| \leq d$. The vector $v$ is then re indexed appropriately and the released station goes back along the communication path to the base station.

We show that both strategies preserve the validity property of the communication path in the following theorem.

Theorem 1. If a communication path is valid then, after applying the Go-ToTHE-MIDDLE strategy, it remains valid.

\section{Static setting}

We analyze the convergence rate of Go-To-THE-MidDLE and assume a static scenario - the explorer does not move. We measure the time which is required 
so that every relay station is within some given distance from the straight line connecting the base station and the explorer.

For the purpose of this analysis we assume that the number of nodes on the path is $n$. We do not remove any nodes from the path, even if they are very close to each other. The positions of nodes $v_{1}$ and $v_{n}$ are fixed - they do not move during the execution of Go-TO-THE-MIDDLE, while all other nodes can move. This corresponds to an explorer standing at its position, and all relay stations executing the Go-To-THE-MIDDLE strategy.

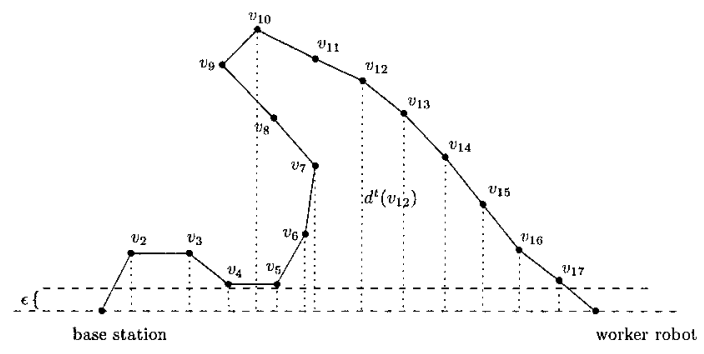

Fig. 2. Relay stations and the area of diameter $\varepsilon$ around the straight line

For a node $v_{i}$ we define $d^{t}\left(v_{i}\right)$ to be the distance of node $v_{i}$ to the straight line crossing nodes $v_{1}$ and $v_{n}$ before step $t$ of the execution GO-TO-THE-MIDDLE. Distance of a point to a line is defined in the usual geometrical way, as depicted in Fig. 2. We assume that at the beginning all nodes (relay stations) are on one side of the line connecting the explorer and the base station. If not, the nodes can be divided into distinct segments, and the analysis can be applied in each segment separately. The case, when all nodes are on one side yields the worst case.

Theorem 2 (Main Theorem). Consider a valid communication path with $n-2$ relay stations. Then after at most $9 n^{2} \log \frac{1}{\varepsilon} n$ steps for every $i$ it holds $d\left(v_{i}\right) \leq \varepsilon$ for any $\varepsilon>0$.

Proof. Obviously it holds $d^{t}\left(v_{1}\right)=d^{t}\left(v_{n}\right)=0$ for all $t \geq 1$. We define $A^{t}:=$ $\left[d^{t}\left(v_{2}\right) \ldots, d^{t}\left(v_{n-1}\right)\right]$ to be the vector of distances of relay stations to the straight line. $A^{0}$ describes the start configuration.

Then after one step of Go-To-THE-MIDDLE the distance

$$
d^{t}\left(v_{i}\right)=\frac{d^{t-1}\left(v_{i-1}\right)+d^{t-1}\left(v_{i+1}\right)}{2}
$$

for all $1<i<n$, which effectively means that $d^{t}\left(v_{2}\right)=d^{t-1}\left(v_{3}\right) / 2$ and $d^{t}\left(v_{n-1}\right)=d^{t}\left(v_{n-2}\right) / 2$ since $d^{t}\left(v_{1}\right)=d^{t}\left(v_{n}\right)=0$.

We can describe the changes of the vector $A^{t}$ by multiplying it with an appropriate transition matrix $L$ so that $A^{t}=A^{t-1} L=A^{0} L^{t}$. This $n \times n$ matrix 
is defined as follows: $L(i, j)=\frac{1}{2}$ for all $i, j$ such that $|i-j|=1$. For all other $i, j$ we have $L(i, j)=0$.

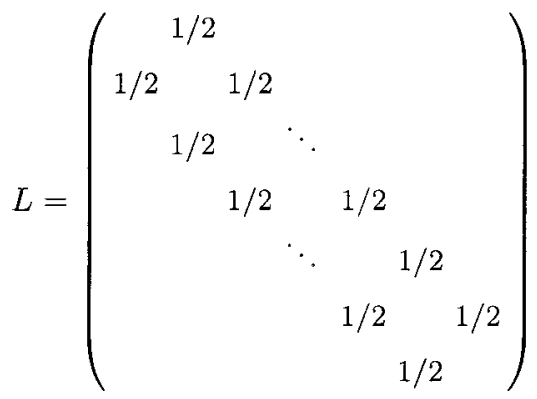

Matrix $L$ is symmetric, substochastic and irreducible. By Lemma 1 all eigenvalues of $L$ are different and thus $L$ is diagonalizable. The rest of the proof of the Main Theorem goes in the following way. We will compute the eigenvalues and eigenvectors of $L$. A lemma about the convergence rate of $L^{t}$ will allow us to give an upper bound on the largest value of $L^{t}$ after $t$ steps. From this we will conclude a bound on the largest value of $A^{t}$ and the Main Theorem easily.

Lemma 1. The eigenvalues of the matrix $L$ are

$$
\lambda_{j}=\cos \left(\frac{j \pi}{n+1}\right), \quad j=1, \ldots n .
$$

The corresponding eigenvectors are

$$
x_{j}(i)=\sin \left(\frac{\pi j i}{n+1}\right), \quad i=1, \ldots, n, \quad j=1, \ldots, n .
$$

Lemma 2. For a diagonalizable, irreducible, symmetric, substochastic $n \times n$ matrix $P$ and any $i, j$ we have

$$
P^{k}(i, j) \leq n \alpha \beta^{k}
$$

where $\beta$ is the largest absolute value of eigenvalues of the matrix $P$ and $\alpha=$ $\max _{i, j, i^{\prime}, j^{\prime}}\left|x_{j}(i) \cdot x_{j^{\prime}}\left(i^{\prime}\right)\right|$ with $x_{j}$ denoting the $j$-th eigenvector of matrix $P$.

The proofs of both lemmas can be found in the full version of the paper. After $t$ steps, we have $L^{t}(i, j) \leq n \alpha \beta^{t}$ for any $i, j$, sticking to the definitions of $\alpha$ and $\beta$ from Lemma 2. As all entries of all eigenvectors of $L$ are not larger than 1 , we have $\alpha \leq 1$. The value of $\left|\cos \frac{j \pi}{n+1}\right|$ is the largest for $j /(n+1)$ approaching 0 or 1 . Without loss of generality we set $j=1$. Then we have $\beta=\cos \frac{\pi}{n+1}$.

Now assuming that the communication distance between nodes is $d$, we know that $A^{0}$ can contain an entry as big as $d n$. On the other hand we know that entries of $L^{t}$ are always non-negative. Recall that $A^{t}=A^{0} L^{t}$. Then to have $d^{t}\left(v_{i}\right) \leq \varepsilon$ we must have each element of $L^{t}$ smaller than $\frac{\varepsilon}{d n^{2}}$ since $d^{0}\left(v_{i}\right) \leq d n$. 
We thus have to find a $t$ such that $L^{t}(i, j) \leq \frac{\varepsilon}{d n^{2}}$ for all $i, j$. Using Lemma 2 we should then have $n \alpha \beta^{t} \leq \frac{\varepsilon}{d n^{2}}$ and accordingly $\beta^{t} \leq \frac{\varepsilon}{d n^{3}}$. We still have to find an upper bound on $\beta$. As argued before, $\beta$ is largest, when $\pi /(n+1)$ approaches 0 . Thus let us expand $\cos x$ around $x=0$ from the Taylor series. We obtain $\cos x \leq 1-\frac{x^{2}}{2}+\frac{x^{4}}{24}$, and set $x=\pi /(n+1)$. Since $\pi^{2} / 2 \geq 1$ and $\pi^{4} / 24 \leq 5$ we obtain

$$
\begin{aligned}
\cos \frac{\pi}{n+1} & \leq 1-\frac{\pi^{2}}{2(n+1)^{2}}+\frac{\pi^{4}}{24(n+1)^{4}} \\
& \leq 1-\frac{1}{(n+1)^{2}}+5 \frac{1}{(n+1)^{4}}
\end{aligned}
$$

Since $\frac{5}{(n+1)^{4}} \leq \frac{1}{2(n+1)^{2}}$ for a sufficiently large $n$ we have $\cos \frac{\pi}{n+1} \leq 1-\frac{1}{2(n+1)^{2}}$. This lets us conclude that for $t=2(n+1)^{2}$ we obtain $\beta^{t} \leq 1 / e$ and for $t=$ $2(n+1)^{2} \cdot \ln \frac{1}{\varepsilon} d n^{3}$ we get $\beta^{t} \leq \frac{\varepsilon}{d n^{3}}$. Assuming that $d$ is constant and upper bounding $2(n+1)$ with $3 n$ we have that $\beta^{t} \leq \frac{\varepsilon}{d n^{3}}$ for $t=9 n^{2} \ln \frac{1}{\varepsilon} n$. This proves that after $t=9 n^{2} \ln \frac{1}{\varepsilon} n$ steps we have $d^{t}\left(v_{i}\right) \leq \varepsilon$.

\section{Dynamic setting}

In this section we investigate the performance of the Go-To-THE-MIDDLE strategy in a dynamic scenario. We first present a route for the explorer which apparently is a hard instance for our strategy. In the second part we investigate our strategy on a very regularly winding route, and on a random walk.

\subsection{A hard case}

We set the maximum transmission distance of stations to 5 units. The experiment starts with the explorer in distance $r$ from the base station. Relay stations are aligned on a straight line between the base station and the explorer, each of them in distance 5 from its neighbors.

Then the explorer starts to walk on a circle with radius $r$ around the base station. The relay station path has to keep up with the motion of the explorer. We let the explorer move always with the same constant speed of 1 unit per time step.

We have discovered that direction changes are profitable for the Go-TOTHE-MIDdLE strategy - see for example the experiments in the next subsection. According to this observation a cyclic scenario is very hard for the Go-ToTHE-MIDdLE - since the explorer steadily moves on the circle, it does not meaningfully change its movement direction and has a high angular speed.

We can alter the speed of the relay stations by allowing them to execute a variable number of GO-TO-THE-MIDDLE rounds per time unit. We denote the number of Go-TO-THE-MIDDLE rounds per time unit as the speed of the relay stations. 


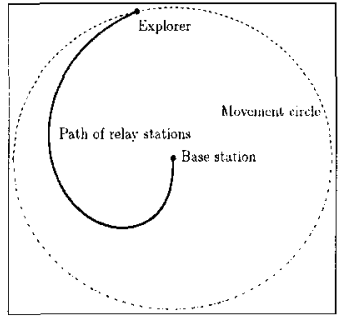

(a) Path of relay stations following explorer

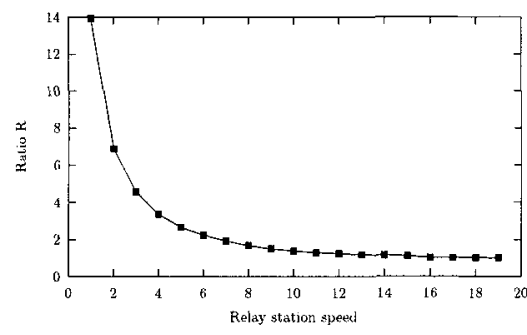

(b) Ratio $\mathcal{R}$ in dependence of relay station speed

Fig. 3. Go-To-ThE-MiddLe for the hard movement model

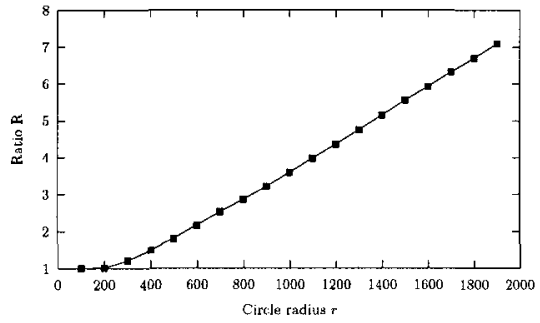

(a) Ratio $\mathcal{R}$ in dependence of radius

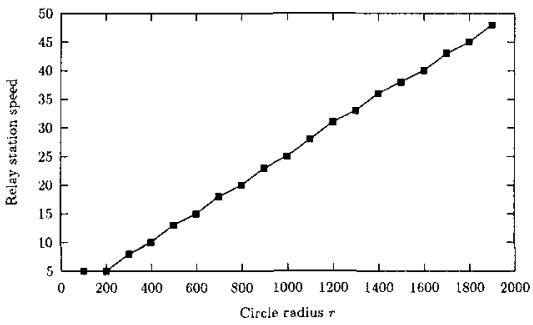

(b) Speed necessary for a ratio $\mathcal{R} \leq 1.5$

Fig. 4. Performance of Go-To-The-Midde in the hard movement model

The performance of the GO-To-THE-MIDDLE strategy is measured in terms of the length of the communication path between the explorer and the base station. In an optimal solution, this length would be always equal to $r$. Since the relay stations may not keep up with the explorer, the length of the line may increase to more than $r$ (obviously new relay stations are introduced then).

We observe that for each radius $r$ and each speed $s$ there is some length $l_{\text {max }}$ of the communication line which is stable, i.e. the communication line length won't exceed this value no matter how long the experiment runs. Figure 3(a) shows the typical curve of the communication line after it reached its stability point. This curve will now only rotate with the movement of the explorer on the circle.

To visualize the performance of the strategy we introduce the ratio $\mathcal{R}$ between the length of the communication line $l_{\max }$ and the length of the optimal interval connecting the explorer and base station. This ratio is investigated in Fig. 3(b) for different speeds of relay stations. Fig. 4(b) shows the ratio $\mathcal{R}$ in dependence of the radius $r$ with the speed fixed to 10 for all radii. 


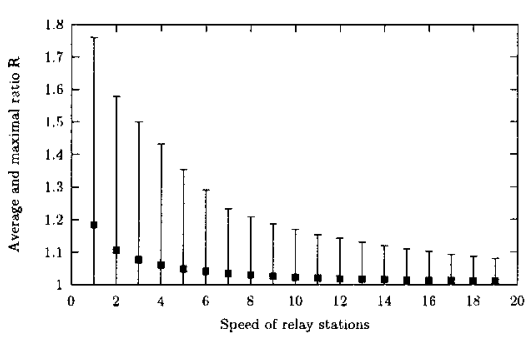

(a) Ratio $\mathcal{R}$ for random walk in dependence of speed

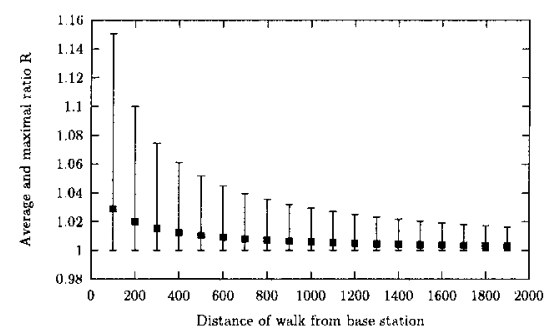

(c) Ratio $\mathcal{R}$ for random walk in dependence of distance

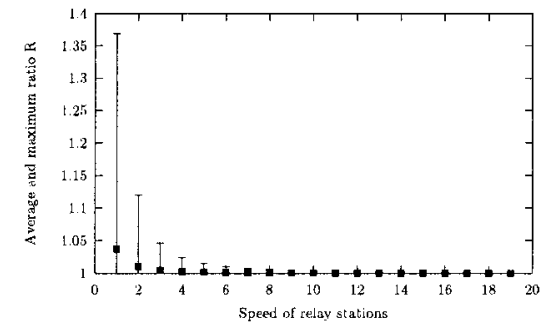

(b) Ratio $\mathcal{R}$ for snake-like exploration in dependence of speed

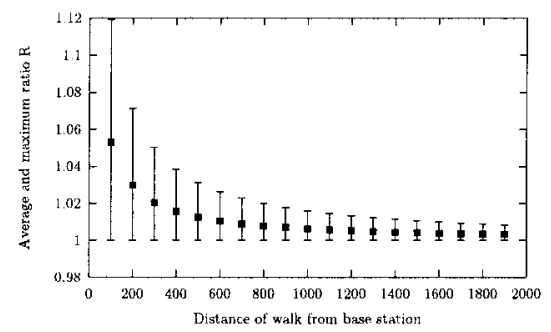

(d) Ratio $\mathcal{R}$ for snake-like exploration in dependence of distance

Fig. 5. Performance of Go-To-THE-Middle for average-case movement models

The maximum length of the communication path $l_{\max }$ (and thus the ratio $\mathcal{R}$ ) grows with the radius $r$, since with the radius $r$ the number of employed relay stations grows and the propagation of the explorer's position updates takes longer time. The growth of $\mathcal{R}$ is linear with the radius.

Fig. 4(b) shows the speed necessary for the ratio $\mathcal{R}$ to be not greater than 1.5. This calculated speed is thus necessary to have a communication path which is a fairly well approximation of the optimal one. We also see a linear increase here.

\subsection{Average cases}

Within this section we investigate two movement models for the explorer. One of them is a random walk on the plane, performed with a constant speed, with a direction randomly chosen in each time step. The direction is chosen uniformly at random from the angle $(-30,+30)$ degrees from the current direction of the explorer. The movement patterns are depicted in the full version of the paper.

For both movement models the ratio between the optimal communication path length and the actual communication path length $\mathcal{R}$ has been computed in each time step. Figures 5(a) and (b) show the average and maximum values of this ratio for both movement models and for different speeds. The same movement pattern can be executed in various distances from the base station. 
Figure 5(c) and (d) shows the results. Interestingly, when the movements are performed in a large distance from the base station, the ratio between the optimal communication path length and the actual path length is low - this comes from the fact that a small movement of the explorer in a large distance from the base stations does not cause large changes in the position of the optimal path and the angular speed of the explorer is low.

\section{Conclusion}

The experiments and theoretical considerations regarding the performance of Go-TO-THE-MIDDLE allow to compute a relay station speed which will give a good approximation factor of the optimal communication path by the relay stations.

The experimental analysis has been performed to obtain information on the behavior of our strategy in various situation. This analysis gives several hints on how to choose the speed of relay stations when the movement pattern of the explorer is not known beforehand. The experimental average-case analysis proves that our strategy can effectively maintain the communication path within an approximation factor of 1.5 with a relay station speed as low as 3 , when certain assumptions about the movement of the explorer are known.

\section{References}

1. Y. U. Cao, A. S. Fukunaga, and A. B. Kahng. Cooperative mobile robotics: Antecedents and directions. In Autonomous Robots, volume 4, pages 1-23, 1997.

2. C. W. Reynolds. Flocks, herds, and schools: A distributed behavioral model. In Computer Graphics, pages 25-34, 1987.

3. Y. Liu and K. M. Passino. Swarm intelligence: Literature overview.

4. R. Arkin. Behavior-based robotics. Cambridge, MA: MIT Press, 1998.

5. G. Dudek, M. Jenkin, E. Milios, and D. Wilkes. A taxonomy for swarm robotics. In IEEE/TSJ International Conference on Intelligent Robots and Systems, pages 441-447, 1993.

6. K. Sugihara and I. Suzuki. Distributed motion coordination of multiple mobile robots. In 5th IEEE International Symposium on Intelligent Control, volume 1, pages $138-143,1990$.

7. Ichiro Suzuki and Masafumi Yamashita. Distributed anonymous mobile robots: Formation of geometric patterns. SIAM J. Comput., 28(4):1347-1363, 1999.

8. I. Chatzigiannakis, M. Markou, and S. Nikoletseas. Distributed circle formation for anonymous oblivious robots. In LNCS, volume 3059, pages 159-174, 2004.

9. Q. Chen and J. Y. S. Luh. Coordination and control of a group of small mobile robots. In IEEE International Conference on Robotics and Automation, volume 3, pages $2315-2320,1994$.

10. H. Ando, Y. Oasa, I. Suzuki, and M. Yamashita. Distributed memoryless point convergence algorithm for mobile robots with limited visibility. In IEEE Transactions on Robotics and Automation, volume 15, 1999. 